
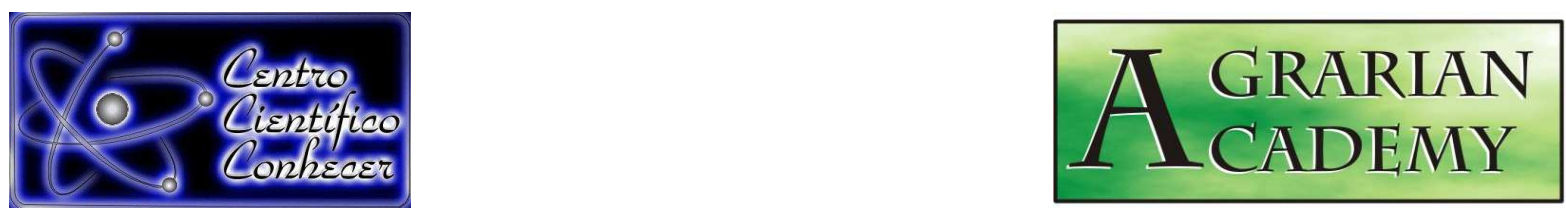

\title{
ESTIMATIVAS DA ÁREA FOLIAR DE ABIEIROS (Pouteria caimito) POR MEIO DE MODELOS ALOMÉTRICOS
}

Samuel Ferreira da Silva ${ }^{1}$, Lucas Rosa Pereira ${ }^{2}$, Patricia Alvarez Cabanez ${ }^{2}$, José Augusto Teixeira do Amaral $^{3}$

1.Doutorando em Produção Vegetal da Universidade Federal do Espírito Santo

(UFES), Alegre - ES, Brasil (samuelfd.silva@yahoo.com.br).

2.Mestrando(a) em Produção Vegetal da Universidade Federal do Espírito Santo (UFES), Alegre - ES, Brasil.

3.Professor Doutor da Universidade Federal do Espírito Santo (UFES), Alegre - ES, Brasil.

Recebido em: 03/01/2014 - Aprovado em: 04/04/2014 - Publicado em: 12/04/2014

\section{RESUMO}

O objetivo deste trabalho foi testar e obter o melhor modelo matemático para estimativa da área foliar do abieiro (Pouteria caimito) em função das suas dimensões alométricas. Utilizou-se um pomar localizado na propriedade São Domingos, no município de Alegre, Sul do Estado do Espírito Santo, onde foram coletadas 80 folhas de 10 abieiros em janeiro de 2014. As regressões foram determinadas considerando-se a área foliar real como variável dependente e o comprimento $(\mathrm{C})$, largura $(\mathrm{L})$ e o produto do $(\mathrm{C} \times \mathrm{L})$ de cada folha como variáveis independentes. Com base nos resultados obtidos, conclui-se que a equação polinomial $y=-0,0025 x^{2}+$ $1,0257 x$ - 5,9942 foi o melhor modelo matemático para estimar a área foliar do abieiro, com $\mathrm{R}^{2}$ de 0,84 . Portanto, os modelos que utilizam o produto das dimensões lineares $(C \times L)$ são os mais adequados para estimar a área das folhas do abieiro, uma vez que apresentam maior correlação.

PALAVRAS-CHAVE: dimensionamento foliar, modelos matemáticos, Pouteria caimito

\section{ESTIMATES OF LEAF AREA ABIEIROS (Pouteria caimito) THROUGH ALLOMETRIC MODELS}

\section{ABSTRACT}

The objective of this study was to test and get the best mathematical model to estimate leaf area abieiro (Pouteria caimito) according to their dimensions Allometric. We used an orchard located in São Domingos property in the municipality of Alegre, southern Espírito Santo, which were collected 80 sheets of 10 abieiros in January 2014. The regressions were determined considering the actual leaf area as the dependent variable and the length $(L)$, width $(W)$ and the product $(L \times W)$ of each sheet as independent variables. Based on these results, we conclude that the polynomial equation $y=-0.0025 x^{2}+1.0257 x-5.9942$ was the best mathematical model to estimate leaf area abieiro with $\mathrm{R}^{2}$ of 0.84 . Therefore, models that use the 
product of the linear dimensions $(\mathrm{L} \times \mathrm{W})$ are best suited to estimate the leaf area of abieiro, since the highest correlation.

KEYWORDS: Pouteria caimito, leaf design, mathematical models.

\section{INTRODUÇÃO}

O abieiro (Pouteria caimito) é uma espécie amazônica, pertencente à família das sapotáceas, é uma árvore de cinco a seis metros de altura, com folhas pecioladas, glabras e os frutos possuem forma ovóide ou esférica com casca amarela e lisa, com massa entre 300 e 1000 gramas, polpa translúcida, branca ou amarela, mucilaginosa, doce ou insípida, contendo de uma a cinco sementes grandes e lisas de cor preta e com grande aceitação popular, utilizado em sua maioria na forma in natura (DONADIO et al., 1992).

MARACAJÁ et al. (2008) salientam que a área foliar é uma das mais importantes medidas de avaliação do crescimento vegetal, por estar ligada ao incremento de matéria seca nas plantas, logo, sua estimativa é de suma importância, uma vez que a arquitetura da copa e os efeitos da interceptação da radiação solar pela folhagem interferem na produção e na composição nutricional dos frutos.

Vale salientar que o conhecimento da área foliar permite a estimativa da perda de água por transpiração, devido às folhas serem os principais órgãos responsáveis pelas trocas gasosas entre a planta e o ambiente, e pelo processo fotossintético que depende da absorção da energia luminosa e sua conversão em energia química (FAVARIN et al., 2002; TAIZ \& ZEIGER, 2013).

Ainda segundo esses autores a área foliar pode ser considerada um dos parâmetros indicativos de produtividade, sendo suas medições essenciais para entender a interação entre o crescimento da planta e o ambiente.

Com o intuito de facilitar o processo de medição da área foliar de diversas culturas, utilizam-se equações matemáticas, sendo este, um método fácil e rápido de ser executado (AMARAL et al., 2009).

Os modelos matemáticos envolvem medições lineares, tais como comprimento foliar ou largura foliar, ou alguma combinação dessas variáveis, geralmente, têm boa precisão para estimar a área foliar real, sendo acessíveis em termos de custo, pois é preciso apenas uma régua graduada para se medir as dimensões foliares (BLANCO \& FOLEGATTI, 2005; AMARAL et al., 2009).

Foram desenvolvidas equações que relacionam o comprimento, a largura da folha, ou ambos, para algumas culturas, como o café (FAVARIN et al., 2002), feijãovagem (QUEIROGA et al., 2003), algodão (MONTEIRO et al., 2005), girassol (ROUPHAEL et al., 2007), manga (LIMA et al., 2012), dentre outras (MARACAJÁ et al., 2008; FAGUNDES et al., 2009), com alto grau de precisão.

No entanto, as pesquisas desenvolvidas sobre a estimativa da área foliar do abieiro ainda é um tema em aberto, necessitando de mais estudos com a própria cultura em questão, com diferentes cultivares e diferentes locais de estudo, conforme salientam ALMEIDA et al. (2006), possibilitando melhorar o manejo agronômico desta cultura.

Desta forma, o objetivo deste trabalho foi testar e obter o melhor modelo matemático para estimativa da área foliar do abieiro (Pouteria caimito) em função das suas dimensões alométricas. 


\section{MATERIAL E MÉTODOS}

Em janeiro de 2014, foram coletadas 80 folhas de 10 árvores em um pomar, localizado na propriedade São Domingos no município de Alegre - ES, situado na latitude 204' $50^{\prime \prime} \mathrm{S}$ e longitude $41^{\circ} 32^{\prime} 54^{\prime \prime}$ W e altitude média em torno de $974 \mathrm{~m} \mathrm{e}$ declividade em torno de $22 \%$.

O clima da região segundo a classificação internacional de Köppen é do tipo Cwa, isto é, tropical quente e úmido, com inverno frio e seco, temperatura anual média de $23,1^{\circ} \mathrm{C}$ e precipitação total anual média de $1341 \mathrm{~mm}$ (LIMA et al., 2008).

A definição do número de árvores e folhas amostradas foi baseada em metodologias de estimativa de área foliar adotadas por MONTEIRO et al. (2005), ROUPHAEL et al. (2007) e LIMA et al. (2012) estudando estimativas de área foliar de diferentes culturas. Todas as folhas foram colhidas em árvores que recebiam os mesmos tratamentos técnicos. É importante salientar que todas as folhas utilizadas no presente estudo não apresentavam nenhum dano ou ataque de doenças ou pragas.

Logo após a retirada total das folhas, elas foram devidamente contadas. Após a coleta, as folhas foram acondicionadas em sacos plásticos e rapidamente conduzidas ao Laboratório de Fisiologia e Nutrição Mineral de Plantas da Universidade Federal do Espírito Santo - UFES, onde se determinou as dimensões, o maior comprimento (C) e a maior largura (L) do limbo foliar com o uso de uma régua graduada em $\mathrm{mm}$. O comprimento $(\mathrm{C})$ foi medido ao longo da nervura central, que é a distância compreendida entre a base da folha no ponto de inserção do pecíolo até o seu ápice, e a largura (L) considerada na parte mediana da folha. Após a determinação do comprimento e da largura máximas, realizou-se a obtenção da estimativa da área foliar real de cada folha, utilizando-se do medidor Licor Modelo LI3100.

As regressões foram determinadas, considerando-se a área foliar real de cada folha (AFR) como variável dependente e o maior comprimento (C), a maior largura $(L)$ do limbo foliar e o retângulo circunscrito à folha $(C \times L)$, como variáveis independentes.

Para a escolha do modelo matemático na determinação da (AFR), foram considerados a sua simplicidade e o maior coeficiente de determinação $\left(R^{2}\right)$.

\section{RESULTADOS E DISCUSSÃO}

$\mathrm{Na}$ Tabela 1, são apresentados os valores médios relativos ao comprimento (C), largura (L) e o produto do ( $\mathrm{C} \times \mathrm{L})$ do limbo foliar e à área foliar real (AFR), com seus respectivos desvios padrões, bem como as dimensões máximas e mínimas do limbo foliar.

TABELA 1. Média, desvio padrão, valores máximo e mínimo para comprimento (C), largura (L), produto do $(\mathrm{C} \times \mathrm{L})$ e área foliar real (AFR) do abieiro (Pouteria caimito).

\begin{tabular}{ccccc}
\hline Variáveis / unidades & Média & Desvio padrão & Máximo & Mínimo \\
\hline$(\mathrm{C}) \mathrm{cm}$ & 14,48 & 4,00 & 23,40 & 7,00 \\
$(\mathrm{~L}) \mathrm{cm}$ & 5,12 & 1,15 & 8,00 & 2,50 \\
$(\mathrm{C} \times \mathrm{L}) \mathrm{cm}^{2}$ & 198,57 & 110,96 & 460,20 & 5,00 \\
$(\mathrm{AFR}) \mathrm{cm}^{2}$ & 55,40 & 23,56 & 122,26 & 16,45 \\
\hline
\end{tabular}

Observa-se alto desvio padrão em relação às médias obtidas para as variáveis estudadas, principalmente para a variável $(C \times L)$, devido à utilização de um número 
grande de folhas de tamanhos variados, o que é percebível quando comparados os valores de máximo e mínimo das variáveis estudadas.

Resultados semelhantes foram obtidos por BIANCO et al. (2002) estudando a área foliar de Cissampelos glaberrima, por RAMOS et al. (2008) estudando a área foliar da pupunheira e por AQUINO et al. (2011) estudando a área foliar de girassol, todos os trabalhos estimaram a área foliar por meio de modelos alométricos.

Diferenças nas dimensões foliares são comuns em aferições de grande quantidade de folhas, resultados obtidos por BORGHEZAN et al. (2010) e QUEIROZ et al. (2013), estimando a área foliar de variedades de videira e espécies florestais, respectivamente, corroboram com os resultados obtidos no presente trabalho.

$\mathrm{Na}$ Tabela 2, estão os modelos de regressão obtidos com os respectivos coeficientes de determinação múltipla $\left(R^{2}\right)$ para a área foliar real em relação às variáveis estudadas.

TABELA 2. Modelos de regressões e os coeficientes de determinação obtidos na estimava da área foliar real (AFR) em função do comprimento $(C)$, largura $(L)$ e produto do $(\mathrm{C} \times \mathrm{L})$ para o abieiro (Pouteria caimito).

\begin{tabular}{cccc}
\hline Variável & Modelo & Equação & $\mathrm{R}^{2}$ \\
\hline \multirow{3}{*}{$(\mathrm{C})$} & Polinomial & $\mathrm{y}=-0,0787 \mathrm{x}^{2}+7,6087 \mathrm{x}-37,015$ & 0,81 \\
& Linear & $\mathrm{y}=5,267 \mathrm{x}-20,864$ & 0,80 \\
& Logarítmica & $\mathrm{y}=72,579 \ln (\mathrm{x})-135,7$ & 0,79 \\
& Exponencial & $\mathrm{y}=11,794 \mathrm{e}^{0,1003 \mathrm{x}}$ & 0,78 \\
\hline \multirow{3}{*}{$(\mathrm{L})$} & Exponencial & $\mathrm{y}=8,4844 \mathrm{e}^{0,3477 \mathrm{x}}$ & 0,78 \\
& Linear & $\mathrm{y}=17,998 \mathrm{x}-36,792$ & 0,77 \\
& Polinomial & $\mathrm{y}=0,2813 \mathrm{x}^{2}+15,11 \mathrm{x}-29,746$ & 0,77 \\
& Logarítmica & $\mathrm{y}=84,259 \ln (\mathrm{x})-79,968$ & 0,74 \\
\hline \multirow{3}{*}{$(\mathrm{C} \times \mathrm{L})$} & Polinomial & $\mathrm{y}=-0,0025 \mathrm{x}^{2}+1,0257 \mathrm{x}-5,9942$ & 0,84 \\
& Linear & $\mathrm{y}=0,5704 \mathrm{x}+10,743$ & 0,80 \\
& Logarítmica & $\mathrm{y}=40,899 \ln (\mathrm{x})-118$ & 0,80 \\
& Exponencial & $\mathrm{y}=22,113 \mathrm{e}^{0,0105 \mathrm{x}}$ & 0,74 \\
\hline
\end{tabular}

O maior valor de $\mathrm{R}^{2}$ foi observado para a regressão polinomial entre a área foliar real e o produto do comprimento pela largura do limbo foliar ( $C \times L)$, indicando ser a equação que permite obter estimativas mais acuradas da área foliar do abieiro (Pouteria caimito). Resultados obtidos por BIANCO et al. (2002) estudando a área foliar de Cissampelos glaberrima, demonstraram que regressões geradas entre a área foliar real e o produto do $(\mathrm{C} \times \mathrm{L})$ possuem melhor representatividade com maior $\mathrm{R}^{2}$, corroborando com os resultados obtidos no presente trabalho.

Nota-se que esta equação apresenta estimativa de $\mathrm{R}^{2}$ de 0,84 , indicando que, da variabilidade total existente na área foliar, $84 \%$ podem ser explicados pela regressão polinomial.

QUEIROZ et al. (2013) estimando a área foliar de espécies florestais evidenciaram que a utilização do produto do $(C \times L)$ possibilitou a obtenção de um melhor coeficiente de determinação, ou seja, maiores valores para o $R^{2}$.

Resultados semelhantes também foram encontrados por MALDANER et al. (2009) ao estudarem modelos de estimativa de área foliar em girassol, em que constataram que a equação polinomial oriunda do produto do $(C \times L)$ possibilitou a obtenção de altos valores de $\mathrm{R}^{2}$.

ZUCOLOTO et al. (2006) estudando a estimativa da área foliar para goiabeira e ALMEIDA et al. (2006) para a gravioleira, também concluíram que os melhores resultados estimados foram obtidos quando foi utilizado o produto entre $(C \times L)$.

AGRARIAN ACADEMY, Centro Científico Conhecer - Goiânia, v.1, n.01; p.205 
JANSSEN \& HEUBERGER (1995) salientam que a regressão entre a área foliar e o produto do $(C \times L)$ indica maior precisão, quando comparado às regressões obtidas com a aferição apenas da largura ou comprimento do limbo foliar, o que é comprovado pelo maior valor de $\mathrm{R}^{2}$.

A Figura 1 apresenta os gráficos de correlação da área foliar pelos modelos de regressões utilizados em relação às variáveis estudadas, comprimento $(\mathrm{C})$, largura $(\mathrm{L})$ e produto do $(\mathrm{C} \times \mathrm{L})$ que apresentaram o melhor $\mathrm{R}^{2}$.
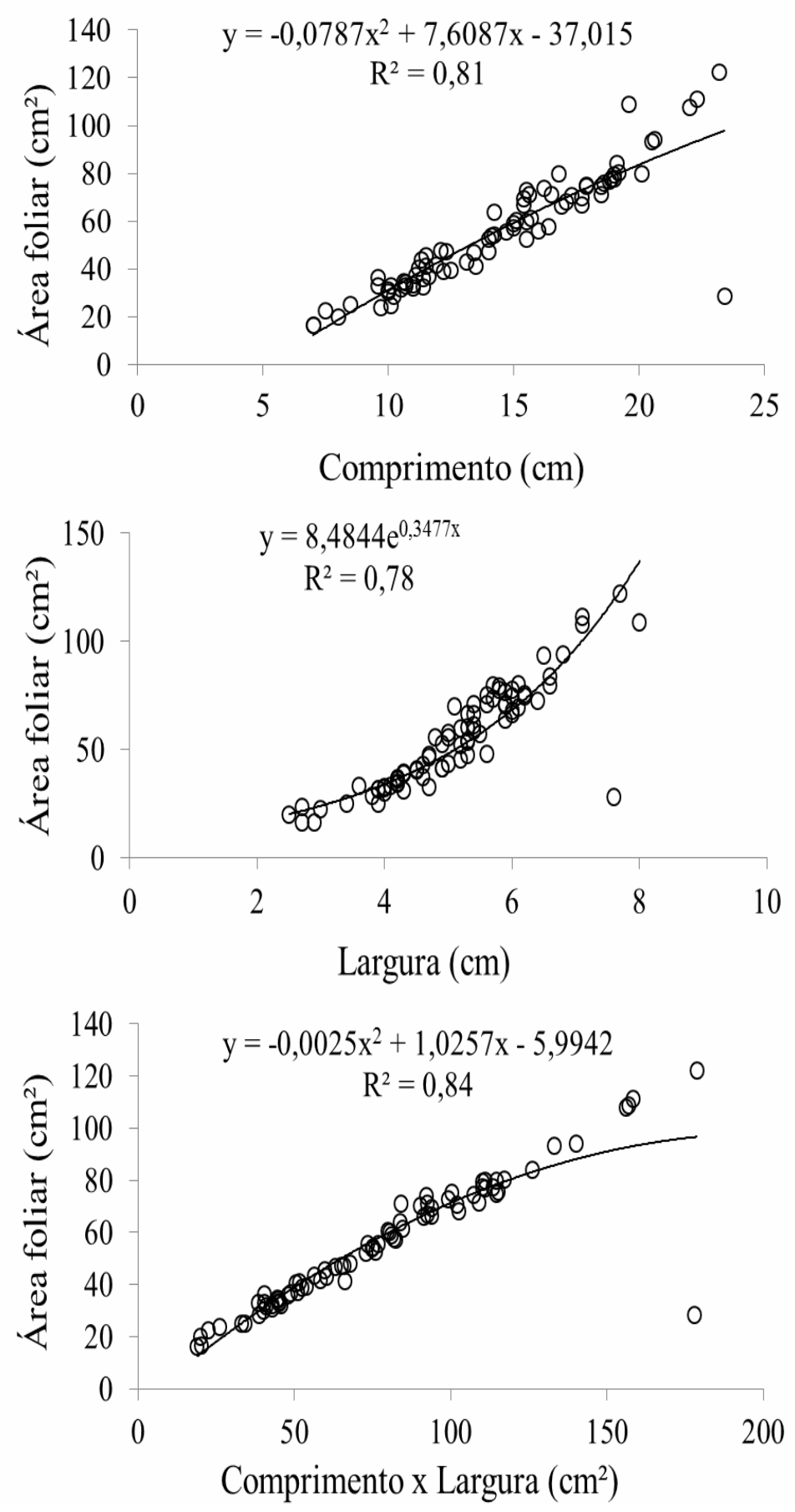

FIGURA 1. Regressões entre a área foliar real e o comprimento $(C)$, largura $(L)$ e o produto do $(C \times L)$. Fonte: os autores. 
Os modelos que utilizam o produto das dimensões lineares $(C \times L)$ são os mais adequados para estimar a área das folhas do abieiro, uma vez que apresentam maior correlação.

De modo geral, apesar de todos os modelos de estimativa de área foliar obtidos, apresentarem valores de $R^{2}$ maiores que 0,74 , sendo estes valores considerados adequados como coeficiente de determinação para a estimativa da área foliar de diversas culturas (ZUCOLOTO et al., 2006; MALDANER et al., 2009 e QUEIROZ et al., 2013) o melhor resultado foi obtido com o modelo polinomial, pois apresentou maior precisão para estimar a área foliar das folhas de abieiro com $\mathrm{R}^{2}$ de 0,84 , considerado como valor adequado no estudo de determinação da área foliar.

\section{CONCLUSÕES}

A equação polinomial $y=-0,0025 x^{2}+1,0257 x-5,9942$ foi o melhor modelo matemático para estimar a área foliar do abieiro (Pouteria caimito) com $\mathrm{R}^{2}$ de 0,84 .

Os modelos que utilizam o produto das dimensões lineares $(C \times L)$ são os mais adequados para estimar a área das folhas do abieiro, uma vez que apresentam maior correlação.

\section{AGRADECIMENTOS}

À Fundação de Amparo à Pesquisa do Espírito Santo - FAPES e à Coordenação de Aperfeiçoamento de Pessoal de Nível Superior - CAPES pela concessão de bolsas aos alunos.

Ao Laboratório de Fisiologia e Nutrição Mineral de Plantas da Universidade Federal do Espírito Santo - UFES pelo apoio estrutural.

\section{REFERÊNCIAS}

ALMEIDA, G. D.; SANTOS, J. G.; ZUCOLOTO, M.; VICENTINI, V. B.; MORAES, W. B.; BREGONCIO, I. S.; COELHO, R. I. Estimativa de área foliar de gravioleira por meio de dimensões foliares do limbo foliar. Revista Univap, v.13 n.24, p.14-20, 2006.

AMARAL, J. A. T.; AMARAL, J. F. T.; SCHILDT, E. R.; COELHO, R. I. Métodos de análise quantitativa do crescimento de plantas. In: FERREIRA et al. (Orgs.). Tópicos especiais em produção vegetal I. Alegre, ES: UFES, Centro de Ciências Agrárias, 2009. p.259-276.

AQUINO, L. A.; SANTOS JÚNIOR, V. C.; GUERRA, J. V. S.; COSTA, M. M. Estimativa da área foliar do girassol por método não destrutivo. Bragantia, v.70, n.4, p.832-836, 2011.

BIANCO, S.; PITELLI, R. A.; CARVALHO, L. B. Estimativa da área foliar de Cissampelos glaberrima usando dimensões lineares do limbo foliar. Planta Daninha, v.20, n.3, p.353-356, 2002.

BLANCO, F. F.; FOLEGATTI, M. V. Estimation of leaf area for greenhouse cucumber by linear measurements under salinity and grafting. Scientia Agrícola, v.62, n.4, p. 305-309, 2005. 
BORGHEZAN, M; GAVIOLI, O; PIT, F. A.; SILVA, A. L. S. Modelos matemáticos para a estimativa da área foliar de variedades de videira a campo (Vitis vinifera $L$.). Ciência e Técnica Vitivinícola, v.25, n. 1, p.1-7, 2010.

DONADIO, L.C.; MARTINS, A.B.G.; VALENTE, J.P. Fruticultura Tropical. Jaboticabal: Funep. 1992. 268p.

FAGUNDES, J. D.; STRECK, N. A.; KRUSE, N. D. Estimativa da área foliar de Aspilia montevidensis (Spreng.) Kuntze utilizando dimensões lineares. Revista Ceres, Viçosa, MG, v.56, n.3, p.266-273, 2009.

FAVARIN, J. L.; DOURADO NETO, D.; GARCÍA Y GARCÍA, A.; VILLA NOVA, N. A.; FAVARIN, M. G. G. V. Equações para a estimativa do índice de área foliar do cafeeiro. Pesquisa Agropecuária Brasileira, Brasília, v.37, n.6, p.769-773, 2002.

JANSSEN, P. H. M.; HEUBERGER, P. S. C. Calibration of process: oriented models. Ecological Modelling, v.83, p.55-56, 1995.

LIMA, J. S. S.; SILVA, S. A.; OLIVEIRA, R. B.; CECÍLIO, R. A.; XAVIER, A. C. Variabilidade temporal da precipitação mensal em Alegre - ES. Revista Ciência Agronômica, v.39, n.2, p.327-332, 2008.

LIMA, R. T.; SOUZA, P. J. O. P.; RODRIGUES, J. C.; LIMA, M. J. A. Modelos para estimativa da área foliar da mangueira utilizando medidas lineares. Revista Brasileira de Fruticultura, v.34, n.4, p.974-980, 2012.

MALDANER, I. C.; HELDWEIN, A.B.; LOOSE, L. H.; LUCAS, D. D. P.; GUSE, F. I.; BERTOLUZZI, M. P. Modelos de determinação não-destrutiva da área foliar em girassol. Ciência Rural, v.39, p.1356-1361, 2009.

MARACAJÁ, P. B.; MADALENA, J. A. S.; ARAÚJO, E.; LIMA, B. G; LINHARES, P. C. F. Estimativa de Área Foliar de Juazeiro por Dimensões Lineares do Limbo Foliar. Revista Verde, v.3, n.4, p.1-5, 2008.

MONTEIRO, J. E. B. A.; SENTELHAS, P. C.; CHIAVEGATO, E. J.; GUISELINI, C.; SANTIAGO, A.V.; PRELA, A. Estimação da área foliar do algodoeiro por meio de dimensões e massa das folhas. Bragantia, v.64, n.1, p.15-24, 2005.

QUEIROGA, J. L.; ROMANO, E. D. U.; SOUZA, J. R. P.; MIGLIORANZA, E. Estimativa da área foliar do feijão-vagem (Phaseolus vulgaris $L$.) por meio da largura máxima do folíolo central. Horticultura Brasileira, v.21, n.1, p.64-68, 2003.

QUEIROZ, J. E.; SILVA, G. H.; SOUZA NETO, A. G. Avaliação estatística da área foliar através de modelos de equações em duas espécies florestais. Revista Verde de Agroecologia e Desenvolvimento Sustentável, v.8, n.1, p.146-153, 2013.

RAMOS, A.; BOVI, M. L. A.; FOLEGATTI, M. V.; DIOTTO, A. V. Estimativas da área foliar e da biomassa aérea da pupunheira por meio de relações alométricas. Horticultura Brasileira, v.26, n.1, p.138-143, 2008. 
ROUPHAEL, Y.; COLLA, G.; FANASCA, S.; KARAM, F. Leaf area estimation of sunflower leaves from simple linear measurements. Photosynthetica, Prague, v.45, n.2, p.306-308, 2007.

TAIZ, L.; ZEIGER, E. Fisiologia vegetal. 5. ed. Porto Alegre: Artmed, 2013. 954p.

ZUCOLOTO, M.; SANTOS, J. G.; BREGONCIO, I. S.; ALMEIDA, G. D.; VICENTINI, V. B.; MORAES, W. B.; COELHO, R. I. Estimativa de área foliar de goiaba por meio de dimensões foliares do limbo foliar. Revista Univap, v.13, n.24, p.1-4, 2006. 